\title{
Bicuspid aortic valve and lusory artery: an unusual association
}

\author{
Luigi Di Martino $\cdot$ Michele Correale $\cdot$ \\ Domenico Cocco • Matteo Di Biase • \\ Natale Daniele Brunetti
}

Received: 7 March 2013/Accepted: 20 April 2013/Published online: 1 May 2013

(c) SIMI 2013

\section{Image}

A patient with bicuspid aortic valve was evaluated in our echocardiography laboratory over serial follow-up controls. Echocardiography showed trivial mitral valve regurgitation and the already known bicuspid aortic valve (Fig. 1a) with a mild regurgitation. An abnormal origin of the subclavian right artery, deriving from the distal aortic arch (Fig. 1b), was unexpectedly found. The patient underwent $\mathrm{CT}$ imaging of the thoracic aorta and supraaortic vessels, which confirmed the right subclavian originating from a lusory artery after a retroesophageal course (Fig. 1c-f). No further vascular abnormalities were found. The caliber of the thoracic aorta was normal as well.

Lusory artery is an uncommon congenital vascular abnormality, whose incidence varies between $0.2-2.5 \%$ [1], resulting from interruption of the fourth right aortic arch between the common carotid artery and subclavian artery and the persistence of the retroesophageal aortic arch [2]. The right subclavian artery thus originates as the last branch of the aortic arch. The condition is usually asymptomatic, even if it may cause dysphagia due to esophageal compression [1]. Complications are

L. D. Martino $\cdot$ M. Correale $(\bowtie) \cdot$ D. Cocco

M. D. Biase - N. D. Brunetti

Department of Cardiology, "Ospedali Riuniti” OO.RR,

University of Foggia, viale L Pinto, 1, 71100 Foggia, Italy

e-mail: opsfco@tin.it described, ranging from atherosclerotic disease and thrombosis [3], potentially eased by the abnormal retroesophageal course, to aneurysma [4] and arterioesophageal fistulae, and other possible serious events usually precipitated by the placement of nasogastric tubes in patients where the erosion of the esophagus was already present [5]. Despite the potentially dangerous implications, this anatomical variant does not usually require surgery, although restoring a normal course of the right subclavian artery by aortic arch repair is feasible and often recommended [1].

The lusory artery is known to be associated with other congenital abnormalities, especially Down syndrome [2]. The association with bicuspid aortic valve appears new.

One of the most consistent findings in bicuspid aortic valve is dilatation of the ascending aorta, even in the absence of clinically significant valvular dysfunction. Dilatation of the ascending aorta represents a key risk factor for dissection and rupture. So, all patients with a bicuspid valve and dilatation of the ascending aorta should have evaluation of the aorta with an MRI or CT angiography. Cardiac MRI is preferred because it also provides the added benefit of information regarding LV function, LV dimensions, and assessment of valve stenosis/regurgitation severity. For those with no aortic dilatation, we tend to use only echocardiography for follow-up.

To our knowledge, there is no any association between lusory artery and persistent branchial cleft cysts.

Further attention should be placed in screening of paucisymptomatic vascular abnormalities in patients found to bear bicuspid aortic valve, so that the need for repair can be determined, according to the risk of potential complications and clinical conditions. 
Fig. 1 Echocardiographyic imaging: a Bicuspid aortic valve; $\mathbf{b}$ Abnormal origin of the subclavian right artery, deriving from the distal aortic arch; CT imaging of the thoracic aorta and supraaortic vessel: c Frontal view of the lusory artery; $\mathbf{d}$ The lusory artery taking origin from the aortic arch and fading to a dorsal plane; $\mathbf{e}$ and $\mathbf{f}$ Coronal view showing the retroesophageal course

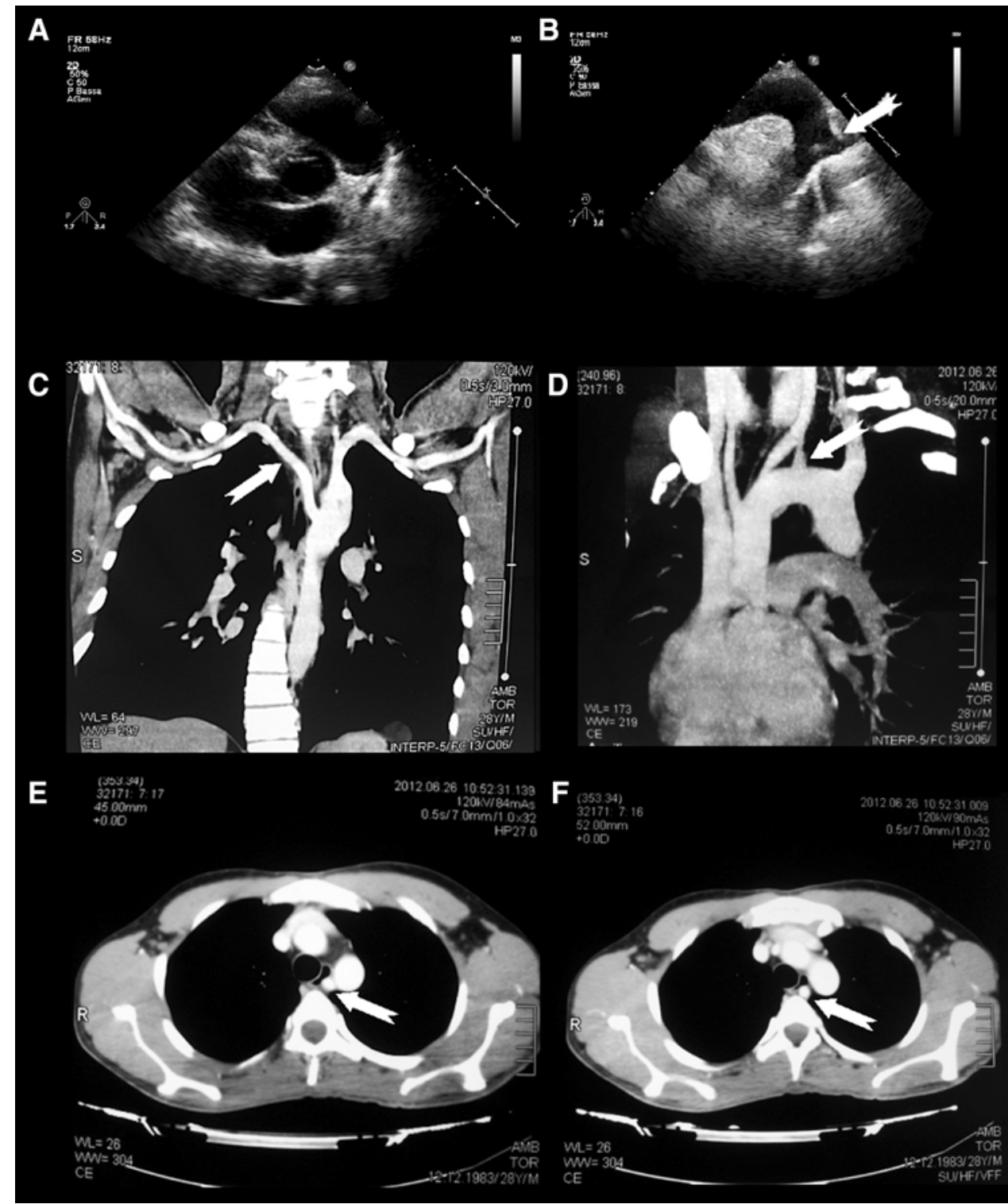

3. Boas N, Desmoucelle F, Bernadet V, Franceschi JC (2002) Rare cause of acute ischemia of the right upper extremity: thrombosis of a retroesophageal subclavian artery. Ann Vasc Surg 16(3):387-390

4. Schmidt FE, Hewitt RL, Flores AA Jr (1980) Aneurism of anomalous right subclavian artery. South Med J 73(2):255-256

5. Feugier P, Lemoine L, Gruner L, Bertin-Maghit M, Rousselet B, Chevalier JM (2003) Arterioesophageal fistula: a rare complication of retroesophageal subclavian arteries. Ann Vasc Surg 17:302-305 